\title{
Magnitude estimates of perceived and remembered length and area
}

\author{
STEPHEN M. KERST and JAMES H. HOWARD, JR. \\ The Catholic University of America, Washington, DC
}

\begin{abstract}
Subjects made repeated magnitude estimates of the lengths of lines or the areas of irregular polygons either from memory or while viewing the stimuli. As had been found previously for cartographic distance and area (Kerst \& Howard, 1978), memory exponents approximated the square of perceptual exponents. Memory exponents differed for length and area, which suggests that more than ordinal information is preserved in memory. These results suggest important similarities between perceptual and memorial processes.
\end{abstract}

Stevens (1975) and others have shown that, for perceptual judgments on additive dimensions such as sound intensity, brightness, and area, the relation between psychological magnitude $(\psi)$ and physical magnitude $(\phi)$ can be described by a power function of the following form:

$$
\psi=\mathbf{k} \phi^{\mathrm{n}}
$$

where $\mathrm{n}$ is a parameter that depends on the judgment continuum and $\mathbf{k}$ is a scaling factor that depends on the units of measurement used. Other work has begun to address the question of whether a similar systematic relation holds between physical and psychological magnitude when judgments are based on an observer's memory for the stimulus (Bjorkman, Lundberg, \& Tarnblom, 1960; Kerst \& Howard, 1978; Moyer, Bradley, \& Cutcomb, 1977; Moyer, Bradley, Sorensen, Whiting, \& Mansfield, 1978). This research has made use of the quantitative methods of perceptual psychophysics to assess the degree to which perceptual information on continuous dimensions is preserved in memory.

For example, Kerst and Howard (1978) asked observers to make magnitude estimates of interstate distances or state areas either while they looked at a map or from memory after they had studied the map for several minutes. Perceptual estimates of distance and area were related to actual stimulus magnitude by power functions whose exponents (.79 for area and 1.04 for distance) were similar to those found for area and line length with conventional procedures (Macmillan, Moschetto, Bialostozky, \& Engel, 1974; Teghtsoonian, 1965). Memory estimates were also related to actual area and distance by power functions with

Since the contribution of the two authors was equivalent, the order of authorship is arbitrary. The authors acknowledge Brian McLaughlin for computer program development, Mari Grant for assistance in data analysis, and James Favret and Martha Sanders for data collection. This research was supported by grants from the National Institutes of Health and the Army Research Institute to Catholic University. Requests for reprints should be addressed to Stephen M. Kerst, School of Education, The Catholic University of America, Washington, DC 20064. exponents of .60 for area and 1.10 for distance. These results suggest that memory for continuous magnitudes is far from crude, but instead preserves much of the quantitative information present in the physical scale; that is, equal stimulus ratios correspond to equal ratios of subjective magnitude estimates at all locations on the scale. Since different memory exponents were found for distance and area, the memory-psychophysical scale for these continua has more than ordinal properties (Stevens, 1975). If memory were based on only ordinal information, then extremely similar memory exponents would be expected for the two continua.

The difference in the two memory exponents has special implications for the relation of perceived and remembered magnitudes. Simple forgetting or lack of confidence in judgments would tend to yield memory exponents that were consistently lower than perceptual ones, since cautious guessing or conservative responding would reduce the range of estimates, and hence reduce the value of the exponent (Teghtsoonian, 1971). Instead, the value of the memory exponent (which reflects the degree of compression or expansion of the range of judgments relative to the range of actual stimulus magnitude) appeared to depend on the value of the perceptual exponent. Memory exponents were approximately equal to the square of the perceptual exponent. In a replication of Kerst and Howard's (1978) Experiment 2, Chew and Richardson (1980), using different materials, that is, a single map including Europe, Africa, and Asia, found exponents of .79 for perception and .64 for memory of cartographic area.

These results were consistent with the working hypothesis (Kerst \& Howard, 1978) that two power functions intervene between the actual physical magnitude of an object and its memorial size estimate. The first function is the typical perceptual one, and the second is introduced during recall. That is, for perception, the psychological magnitude, $\psi \mathrm{p}$, is a power function of the actual physical magnitude, $\phi ; \psi \mathrm{p}=\mathrm{k} \phi^{\mathrm{n}}$. For memory, psychological magnitude, $\psi \mathrm{m}$, is a power function of the previously determined perceptual magnitude, $\psi \mathrm{p} ; \psi \mathrm{m}=\mathrm{k}^{\prime} \psi \mathrm{p}^{\mathrm{n}^{\prime}}$. By substitution it may be shown that memorial judgments are 
also power functions of the actual physical magnitudes: $\psi \mathrm{m}=\mathrm{k}^{\prime}\left(\mathrm{k} \phi^{\mathrm{n}}\right)^{\mathrm{n}^{\prime}}=\mathrm{K} \psi \mathrm{p}^{\mathrm{n} \cdot \mathrm{n}^{\prime}}=\mathrm{K} \phi^{\mathrm{N}}$, where $\mathrm{K}$ is a new scaling factor to accommodate the memory estimates. Furthermore, since the two transformations are assumed to be identical, the memory exponent, $\mathrm{N}$, approximates the square of the perceptual exponent, $n$ (i.e., since $n=n^{\prime}$, $\mathrm{N}=\mathrm{n}^{2}$ ). Using somewhat different procedures, Moyer et al. (1978) independently obtained perceptual and memorial exponents of .87 and .70 , respectively, for judgments of line length. The unexpectedly low perceptual exponent may be due to the absence of a standard in his judgment task (Macmillan et al., 1974). In addition, since Moyer et al. (1978) employed a 24-h retention interval between learning and test, forgetting and cautious judgment may have caused a decrease in the memorial exponent beyond that predicted by our working hypothesis $(.87$ vs. .76). Nevertheless, these results and additional data on state area judgments led Moyer et al. (1978) to suggest a "serial transformation"model similar to the one described above.

To achieve a more satisfactory comparison of perceptual and memorial magnitude estimates, it seemed essential to replicate and extend our previous results in a more conventional magnitude estimation task and with materials other than familiar geographical stimuli. Four aspects of the experiment permitted such a comparison. First, to control for possible contrast effects with perceptual estimates, perceptual stimuli were presented singly with a standard, rather than simultaneously on a map. Second, to minimize the effects of forgetting and confusion on memory estimates, a minimal retention interval and a small number of stimuli (7) were used. Third, to control observers' preexperimental knowledge of the stimuli, horizontal lines and nonsense shapes were used to discourage subjects from basing area estimates on some linear dimension of the figure (Teghtsoonian, 1965). Fourth, for greater stability of estimates, multiple trials were used. In the present study, independent groups of observers made magnitude estimates of the area of irregular shapes or the length of horizontal lines either from memory or while viewing the stimuli.

\section{METHOD}

\section{Subjects}

Students at Catholic University served as subjects and were paid $\$ 3.00$. There were 11 subjects in the shapes memory condition and 12 subjects per group in the shapes perception condition, the lines memory condition, and the lines perception condition.

\section{Apparatus and Stimuli}

All experimental events were controlled by a DEC PDP-11/34 computer. Stimuli were displayed on a Tektronix 4006-1 CRT terminal with a $20 \times 16 \mathrm{~cm}$ screen. The stimuli for length estimates were seven horizontal lines spaced $.6 \ln$ units apart, with a range of $3.6 \ln$ units. The actual lengths used were $.5, .9,1.7,3.1,5.7,10.4$, and $18.9 \mathrm{~cm}$. Each line was centered in the horizontal dimension and was displayed $5 \mathrm{~cm}$ from either the top or the bottom of the screen, with a distance of $6 \mathrm{~cm}$ between the two stimuli. For area estimates, seven computer- generated irregular polygons were used as stimuli. These six-sided shapes were selected from a large set of polygons so that they were spaced an average of $.56 \mathrm{ln}$ units apart in area with a range of 3.35 ln units. ${ }^{1}$ The actual areas used were $1.1,2.3,4.0,6.5,12.6,17.8$, and $31.8 \mathrm{~cm}^{2}$.

\section{Procedure}

Area estimates. All subjects learned to associate a consonant-vowelconsonant (CVC) nonsense syllable of low association value (Stevens, 1951, p. 545) with each of the seven irregular polygons by the anticipation method. Throughout the experiment, a shape was presented either on the right or the left side of the viewing screen, and the side of presentation was counterbalanced within trial blocks. A trial block consisted of seven trials, with each shape being presented once. The order of presentation of the shapes was randomized for each trial block, and CVCs were randomly paired with shapes for each subject. Each shape, paired with its CVC label, was presented individually for $7 \mathrm{sec}$ on the CRT terminal. After this initial phase of training, each shape was displayed alone, and the observer indicated the CVC name for the shape by pressing an appropriately labeled key on the terminal keyboard. After each trial, the correct name was displayed while the shape remained on the screen. The subjects were required to correctly identify the stimuli on three consecutive trial blocks before they began the magnitude estimation task. After training was completed, the shape in the middle of the series was designated as a standard. The subjects in the memory groups made magnitude estimates for each of the remaining six shapes when the nonsense label for each stimulus, the name of the standard, and the value of the modulus were displayed. The subjects in the perceptual group viewed both the standard and the comparison stimulus on each trial in a magnitude estimation procedure. Each shape was centered in the vertical dimension and was presented on either the left or the right side of the screen. The position of the standard (left or right) was counterbalanced within each block of trials. For all subjects, a modulus of 100 was assigned to the standard stimulus for the first of four blocks of magnitude estimation trials. For each remaining block, the modulus was assigned a different value (i.e., 50,80 , or 130 ), and the order of the values was counterbalanced across subjects. On each trial, the value of the modulus for that block of trials was displayed on the screen. Following the magnitude estimation phase of the experiment, recognition memory for the shape stimuli was tested by requiring observers to indicate the correct CVC label for each individually presented stimulus. Two trial blocks were used in this posttest. The subjects were tested individually. Approximately $1 \mathrm{~h}$ was required to complete the entire experiment.

Length estimates. The training and judgment procedures used for length estimates were identical to those described above for area estimates, except that subjects made perceptual or memorial magnitude estimates of the lengths of seven horizontal lines. In the perceptual condition, the position of the standard line (upper or lower) was counterbalanced within each block of trials. As for area estimates, the standard and modulus were presented with the comparison stimuli on each trial in the perceptual condition. In the memory condition, the name of the standard, the value of the modulus, and the CVC label for a line were presented during magnitude estimation trials.

\section{RESULTS}

\section{Psychophysical Analyses}

Five subjects (one in the lines memory condition, one in the shapes perception condition, and three in the shapes memory condition) were dropped from the experiment for failing to follow instructions, that is, for consistently failing to make estimates that increased with increasing stimulus magnitude or failing to base their estimates on the modulus designated for a particular trial block. Power functions were fit to both group and individual estimates for each of the four conditions using linear regression tech- 
Table 1

Power Function Exponents (n) and Correlations (r) for Perceptual and Memorial Estimates of Line Length and Shape Area (Experiment 1)

\begin{tabular}{ccccccc}
\hline & & \multicolumn{2}{c}{ Group Data } & & \multicolumn{2}{c}{ Median Individual Data } \\
\cline { 3 - 4 } \cline { 6 - 7 } & & Perception & Memory & & Perception & Memory \\
\hline \multirow{2}{*}{ Line Length } & $\mathrm{n}$ & .93 & $.84(.86)$ & .90 & $.81(.81)$ \\
& $\mathrm{r}$ & .99 & .99 & .99 & .99 \\
\multirow{2}{*}{ Shape Area } & $\mathrm{n}$ & .77 & $.61(.59)$ & .74 & $.65(.55)$ \\
& $\mathrm{r}$ & .99 & .99 & .99 & .98 \\
\hline
\end{tabular}

Note-Values in parentheses are those predicted by the relation that the memory exponent equals the square of the perceptual exponent.

niques on the log geometric mean estimates and log actual shape areas or line lengths. Exponents (the slopes of the regression lines) and the correlations that describe the goodness of fit of the power functions are shown in Table 1 . The standard was excluded from the analysis, since its value was defined by the modulus provided for each trial block. For both memorial and perceptual data, subjective estimates were systematically related to actual stimulus magnitude. The high correlations obtained from group data and for data from single subjects indicate that this relation is well described by a power function. Comparison of the slopes of the regression lines for group data showed that exponents for length and area judgments differed when estimates were made perceptually $[\mathrm{t}(8)=$ $5.21, \mathrm{p}<.001$, one-tailed test $]$ and when they were based on memory $[t(8)=4.14, p<.005$, one-tailed test]. Since distinct memory exponents are found for the two continua, it appears that the memory-psychophysical scales for length and area have more than ordinal properties (Stevens, 1975).

\section{Compression or Distortion in Memory Estimates}

The hypothesis that memory estimates involve a second power transformation identical to the original perceptual one was tested in the following way. The group geometric mean perceptual estimate for each stimulus was raised to a power equal to the perceptual exponent. These transformed geometric means were then treated as ordinary geometric mean magnitude estimates, and power functions were computed to describe the relationship between these transformed geometric means and the actual stimulus magnitudes. According to the hypothesis of a second power transformation in memory, the exponents of these power functions should not differ from those obtained with actual memory estimates. The results were consistent with this hypothesis. The exponents based on the transformed data did not differ from the actual memory exponent. For the line length, the "transformed exponent" was .87 versus the actual memory exponent of $.84[\mathrm{t}(8)=.64$, $\mathrm{p}>.05$, two-tailed test]. For shape area, the "transformed exponent" was .59 versus the memory exponent of .61 $[\mathrm{t}(8)=.63, \mathrm{p}>.05$, two-tailed test $]$. Since the untrans- formed perceptual exponents differed significantly from memory exponents for area $[t(8)=4.18, p<.005$, onetailed test $]$ and for length $[t(8)=1.87, p<.05$, one-tailed test], the finding of no difference between transformed perceptual and memorial exponents is not due merely to low statistical power. The degree of compression of the range of memory-based judgments relative to the actual stimulus range (as indicated by the exponent) thus appears to be related to the degree of compression or distortion of perceptual judgments for the same continuum. These data indicate that the degree of memory distortion is approximately equal to compounded perceptual distortion.

\section{DISCUSSION}

The present study was carried out to replicate and extend previous work with estimates of geographical area and distance (Kerst \& Howard, 1978) under more controlled conditions. Line length and the area of irregular shapes were used as counterparts to interpoint map distance and map area in a magnitude estimation task. Both the memory and perception estimates were related to actual stimulus values by power functions. The amount of compression or distortion in the range of memory judgments (as indicated by exponents differing from 1.0) depended on the exponent for perceptual judgments. Different memory exponents were found for length and area. This result suggests that subjects have more than rank order information in memory for these dimensions. If memory estimates were based only on ordinal information, this difference in the degree to which the psychological scales are compressed relative to the actual physical scales for the two dimensions would not be expected (Stevens, 1975).

The degree of compression of memory estimates could be quantitatively described as a compounding of the distortion present in perceptual judgments: Memory appeared to systematically exaggerate deviation from veridical estimates. Memory exponents approximated the square perceptual exponents, as was the case for cartographic distance and area (Chew \& Richardson, 1980; Kerst \& Howard, 1978). Similar results have been found recently with distances judged from an artificial map and areas judged from a configuration of circles (Fantini, 1984). One implication of these results is that graphic displays that represent relative quantity by linear extent rather than area will be remembered more accurately.

\section{REFERENCES}

Bjorkman, M., Lundberg, I., \& TARnblom, S. (1960). On the relationship between percept and memory: A psychophysical approach. Scandinavian Journal of Psychology, 1, 136-144.

Chew, E. I., \& Richardson, J. T. E. (1980). The relationship between perceptual and memorial psychophysics. Bulletin of the Psychonomic Society, 16, 25-26.

FANTINI, D. A. (1984, April). Psychophysical functions for size judgments of seen and remembered circles. Paper presented at the annual meeting of the Eastern Psychological Association, Baltimore.

Kerst, S. M., \& Howard, J. H., JR. (1978). Memory psychophysics for visual area and length. Memory \& Cognition, 6, 327-335.

Macmillan, N. A., Moschetto, C. F., Bialostozky, F. M., \& ENGLE, L. (1974). Size judgment: The presence of a standard increases the exponent of the power law. Perception \& Psychophysics, 16, 340-346.

Moyer, R. S., Bradley, D. R., \& Cutcomb, S. (1977, April). Memory psychophysics. Paper presented at the annual meeting of the Eastern Psychological Association, Boston.

Moyer, R. S., Bradley, D. R., Sorensen, M. H., Whiting, J. C., \& MANSFIELD, D. P. (1978). Psychophysical functions for perceived and remembered size. Science, 200, 330-332. 
Stevens, S. S. (Ed.). (1951). Handbook of experimental psychology. New York: Wiley.

Stevens, S. S. (1975). Psychophysics: Introduction to its perceptual, neural, and social prospects. New York: Wiley.

Teghtsoonian, M. (1965). The judgment of size. American Journal of Psychology, 78, 392-402.

Teghtsoonian, R. (1971). On the exponents in Steven's law and the constant in Ekman's law. Psychological Review, 78, 71-80.

\section{NOTE}

1. Due to a programming error, the stimuli were not evenly spaced in log steps. Rather, the spacing between adjacent stimuli was .735 , $.544, .479, .665, .341$, and .584 in natural-log area.

(Manuscript received for publication June 1, 1984.) 